\title{
Influence of bowl shaped substrate holder on growth of polymeric DLC film in a microwave plasma CVD reactor
}

\author{
SAMBITA SAHOO, S K PRADHAN*, VENKATESWARLU BHAVANASI, SWATI S PRADHAN, \\ S N SARANGI ${ }^{\dagger}$ and P K BARHAI ${ }^{\dagger}$ \\ Advanced Materials Technology Department, Institute of Minerals and Materials Technology, Bhubaneswar 751 013, India \\ $\dagger$ Institute of Physics, Bhubaneswar 751 005, India \\ †Department of Applied Physics, Birla Institute of Technology, Mesra 835 215, India
}

MS received 4 September 2011; revised 4 April 2012

\begin{abstract}
The properties of diamond like carbon (DLC) films grown in modified microwave plasma CVD reactor is presented in this paper. By using bowl shaped steel substrate holder in a MW plasma CVD reactor (without ECR), films have been grown at relatively high pressure $(20$ Torr) and at low temperature (without heating). The input microwave power was about $300 \mathrm{~W}$. Earlier, under the same growth conditions, no deposition was achieved when flat molybdenum/steel substrate holders were used. In this study, two different designs of bowl shaped steel substrate holder at different bias have been experimented. Raman spectra confirm the DLC characteristics of the films. FTIR results indicate that the carbon is bonded in the $s p^{3}$ form with hydrogen, and this characteristic is more pronounced when smaller holder is used. UV-visible spectra show high visible transmittance $(\sim 85 \%)$ for films grown in both the holders. The nanoindentation hardness of the films have a wide range, about 4-16 GPa. Field emission scanning electron microscope (FESEM) images reveal that the films have featureless and smooth surface morphology. These films are polymeric in nature with moderately high hardness, which may be useful as anti-scratch and anti-corrosive coatings.
\end{abstract}

Keywords. Diamond like carbon (DLC); polymeric DLC; microwave plasma CVD.

\section{Introduction}

Different forms of carbon such as diamond like carbon (DLC) thin films, carbon clusters (nanotubes and nanodiamonds) and fullerenes have come up with major developments that are expected to find better performing applications in industry. Unlike diamond, DLC can be deposited at room temperature, which finds its applications in the area of magnetic hard disk coatings, wear protective and antireflective coatings for tribological tools and biomedical coatings such as hip implants or stents. These applications are possible due to high hardness and low coefficient friction of DLC films (Neuville 2002; Robertson 2002; Choy 2003; Neuville and Matthews 2007). DLC films have been grown by various methods but the most common technique to grow DLC films is an RF glow discharge system (Holland and Ojha 1979). However, hardly any literature has been reported about the deposition of DLC films by microwave plasma activated CVD (MWPACVD) at high pressure. The major drawback of DLC films is the presence of high residual stress. By applying suitable bias in MWPACVD at high pressure, DLC films with comparatively less residual stress can be grown. Usually MWPACVD is used to grow polycrystalline, nanocrystalline

\footnotetext{
*Author for correspondence (skpradhan@immt.res.in)
}

and epitaxial diamond (Geis et al 1998; Achard et al 2007; Sumant et al 2007) as well as carbon nanotube (Taniguchia et al 2005). Nanocrystalline diamond (NCD) and DLC film grown by plasma assisted CVD process have better quality as compared to other processes.

DLC film grows by the impingement of hydrocarbon species (ions, neutrals) onto the substrate surface. The properties of the growing film are controlled by the energy of the impinging species. The plasma ionization, bias voltage and pressure define the energy of the depositing species. By controlling these parameters, it is possible to tailor films properties. In addition to the above parameters, in a microwave plasma reactor, the cavity size affects the plasma condition and the energy of the depositing species. One way to change or affect the cavity size is by changing the substrate holder shape and size that give edge effect.

The objective of the present work is to study the growth behaviour and quality of the deposited DLC films grown using two different designs of steel substrate holders under different bias conditions. With these objectives, grown films have been characterized for structural, morphological, optical and mechanical properties and are compared. The grown films are found to be polymeric in nature with higher hydrogenated $s p^{3}$ carbon, having smooth surface morphology, moderate hardness, low modulus and high visible transmittance. 


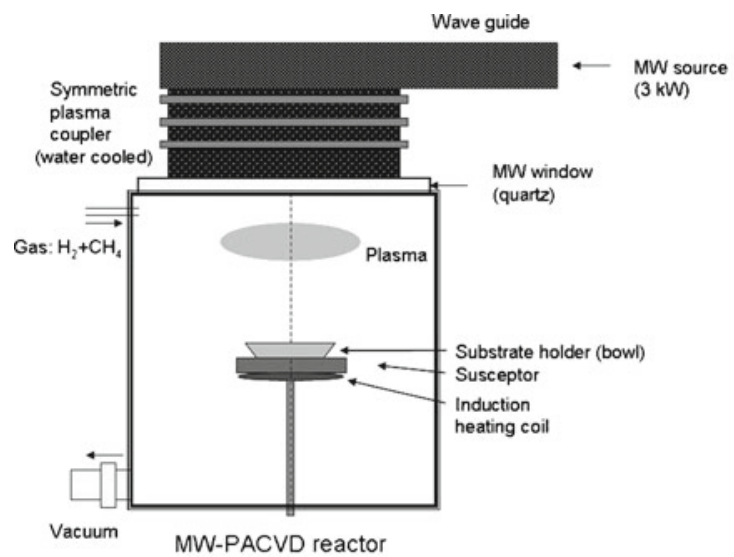

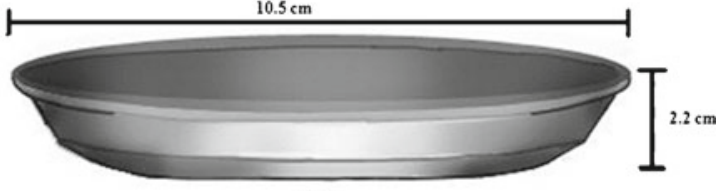

(a)

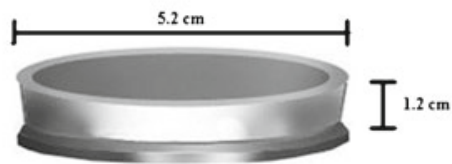

(b)

(c)

Figure 1. Schematic of bowl shaped substrate holders (a) and (b) used for growing DLC films with schematic of MWCVD system (c).
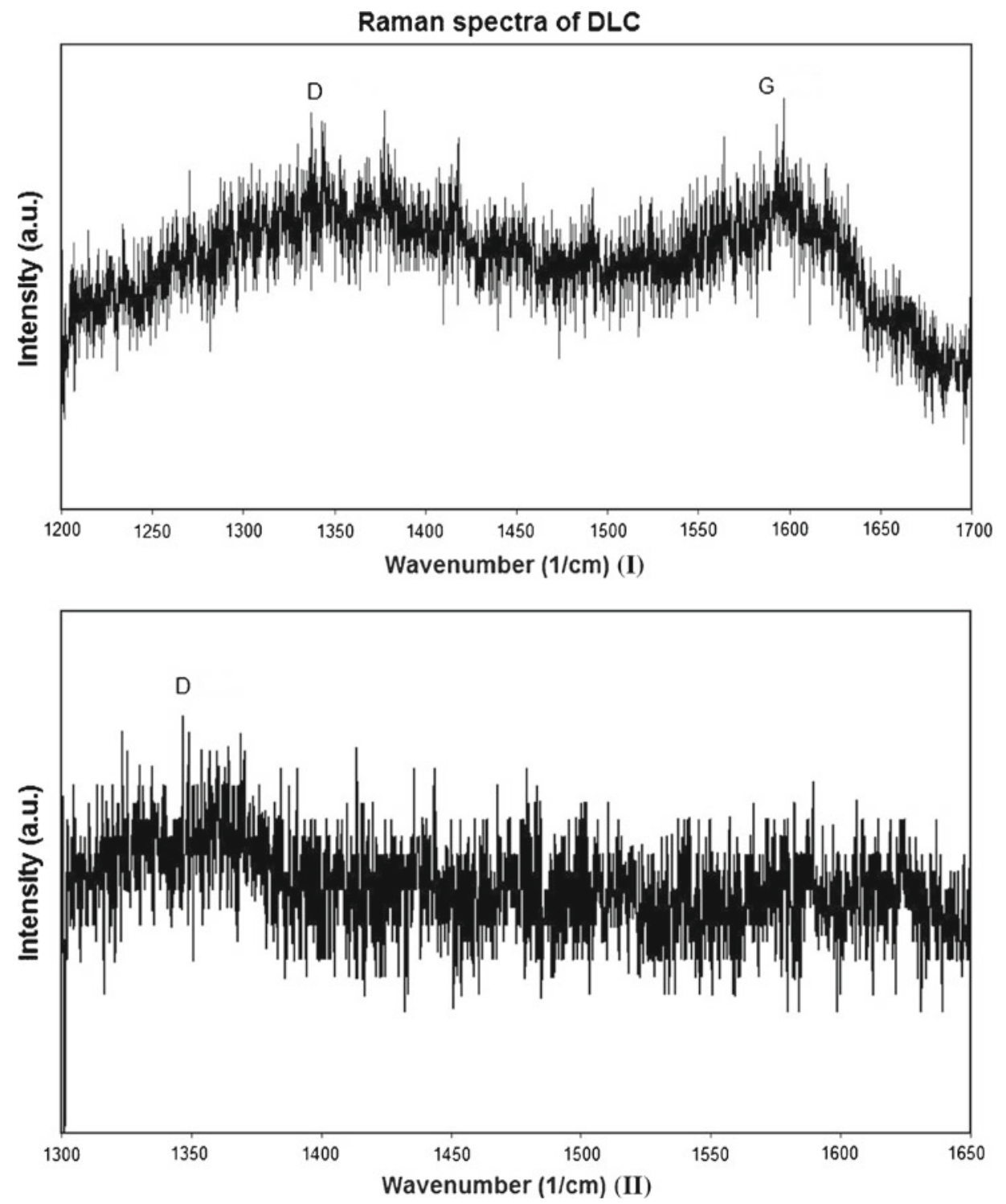

Figure 2. Raman spectra of DLC film grown on (I) holder (a) and (II) holder (b). 


\section{Experimental}

DLC films were grown on silicon (100), quartz and metallic substrates using a $3 \mathrm{~kW}$ MWPACVD system (Seki Technotron, Japan) operated at $300 \mathrm{~W}$. The precursor gases used for deposition of DLC film at room temperature were methane $(2-3 \mathrm{sccm})$, argon $(130-135 \mathrm{sccm})$ and hydrogen (3-4 sccm) varying the applied substrate bias from 0-150 V. Two different designs of bowl shaped steel substrate holder were used. As shown schematically in figure 1, holder (a) had a diameter of $10.5 \mathrm{~cm}$ and holder (b) had a diameter of $5.5 \mathrm{~cm}$. A typical deposition run consisted of substrate loading on to the bowl shaped steel holder and then evacuation of the chamber. After reaching the base vacuum level, argon and hydrogen mixture was introduced and then substrate was heated. Then the plasma was struck and the d.c. bias was adjusted. Plasma cleaning of the substrate was done for $5 \mathrm{~min}$, after that the flow of methane was adjusted and the plasma was maintained at the required power level $(300 \mathrm{~W})$
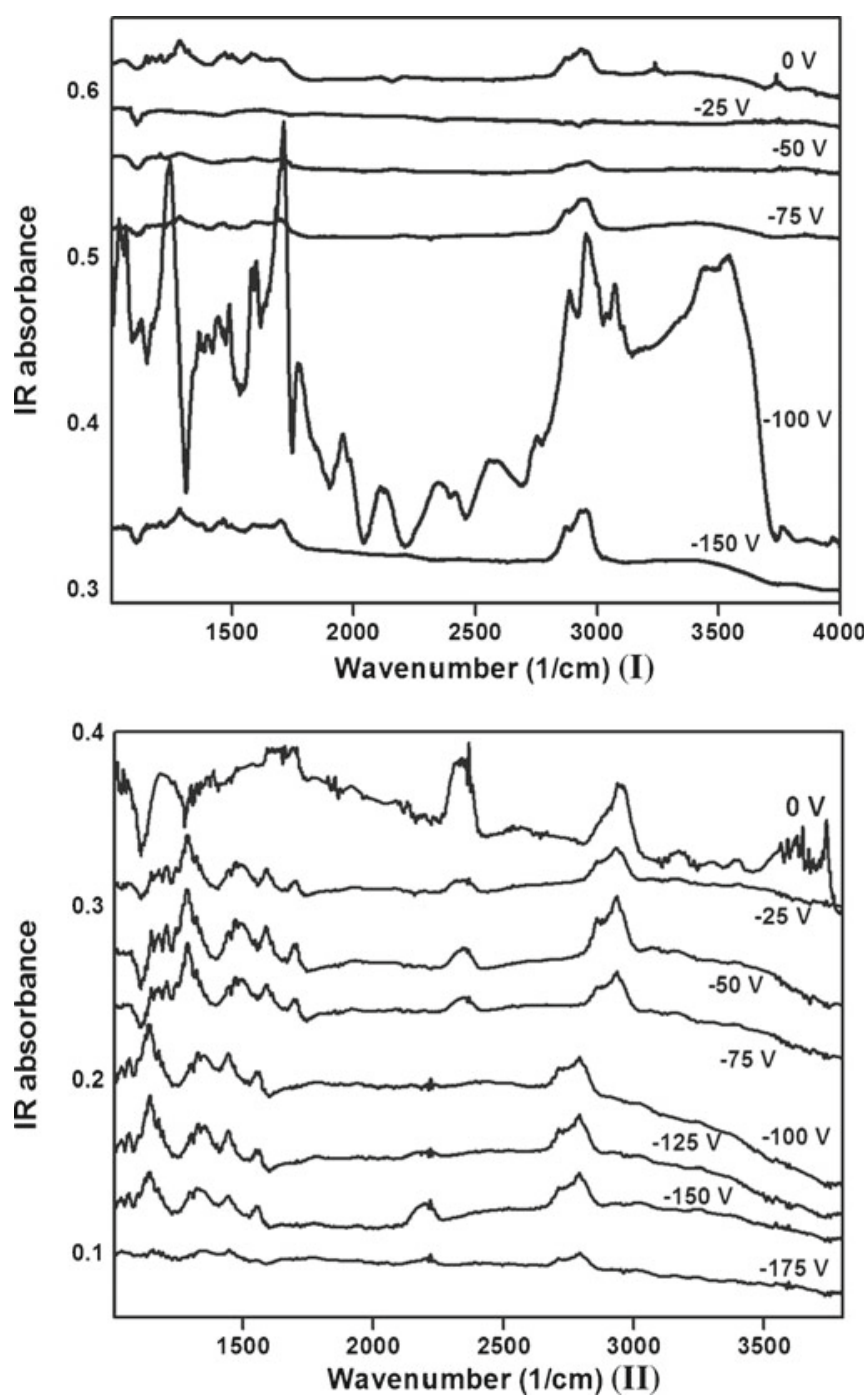

Figure 3. IR absorbance spectra of DLC film deposited at different bias on (I) holder (a) and (II) holder (b). for $2 \mathrm{~h}$. These films were deposited at a relatively high pressure (21 Torr) so that removal of desorbing contaminant species would be easier.

The grown films were characterized by nanoindentation (Fisher-Cripps, UMIS), Raman (Jobin Yvon, U1000), FTIR (Perkin Elmer, spectrum GX), UV-visible spectrometer (Perkin Elmer, lambda 650), and field emission scanning electron microscope (FESEM) (Carl Zeiss, Supra 55).

\section{Results and discussion}

Visible Raman spectroscopy is one of the most useful methods for characterizing DLC films, however, information on $s p^{3}$ bonding cannot be obtained directly because excitation by visible photons at $514 \mathrm{~nm}$ is only sensitive to $s p^{2}$ sites due to their much greater cross-section than that of $s p^{3}$ sites (Sails et al 1996). Nonetheless, much information about the carbon
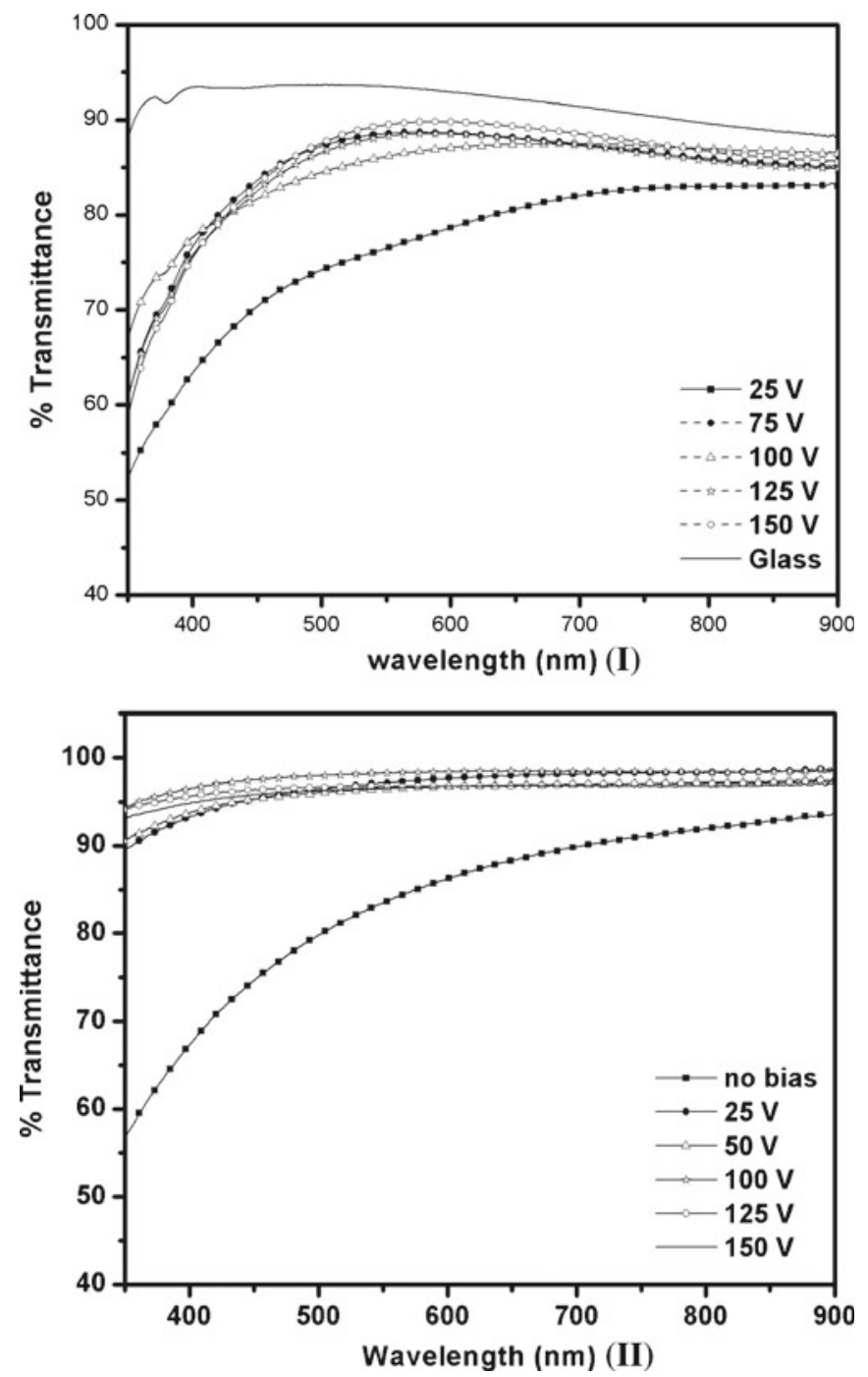

Figure 4. Transmittance spectra (UV-visible) of DLC film in visible and near IR region $(300-800 \mathrm{~nm})$ on (I) holder (a) and (II) holder (b). 

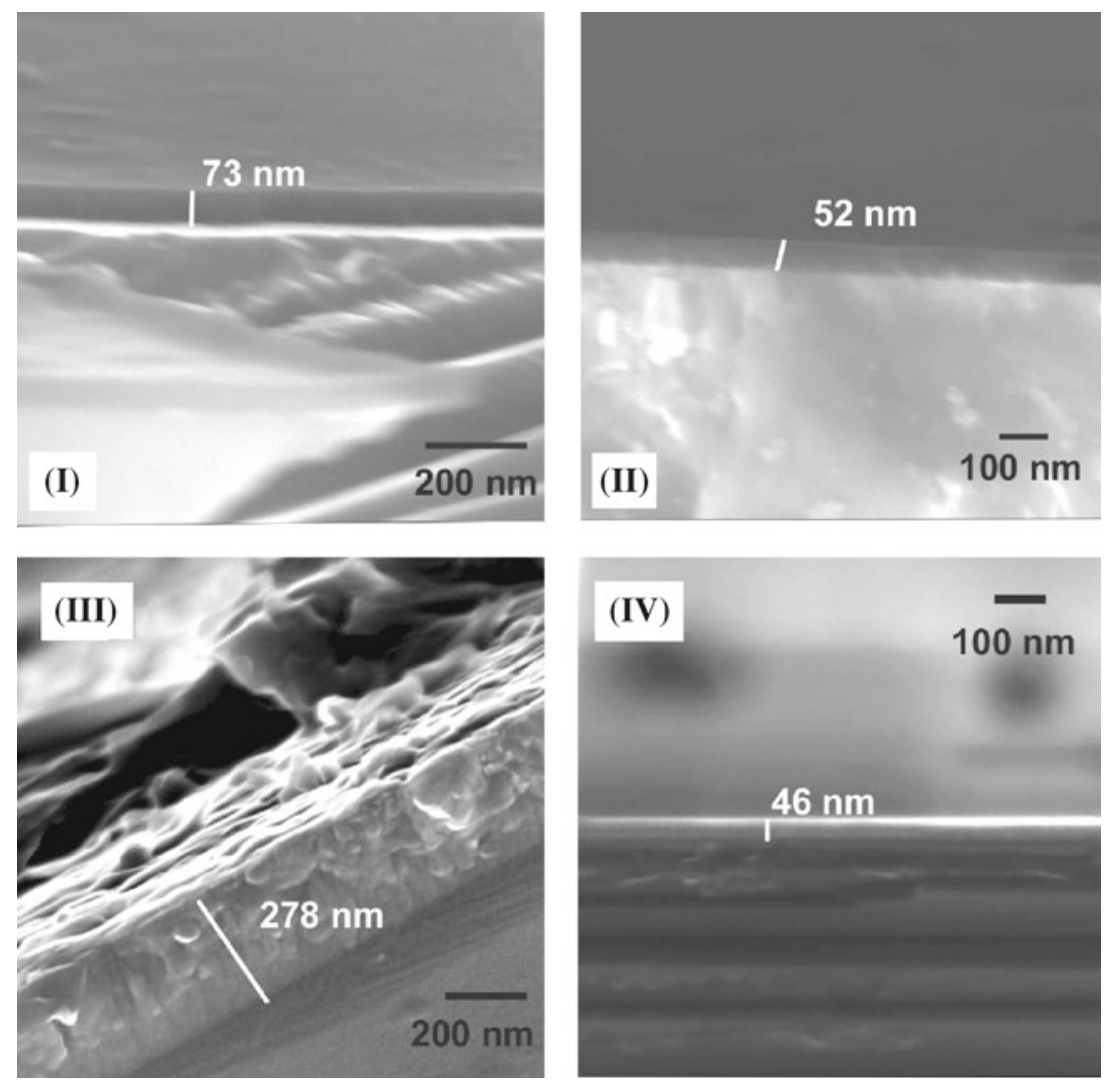

Figure 5. FESEM cross-section images of DLC (I) at $0 \mathrm{~V}$ bias on holder (a), (II) at $-100 \mathrm{~V}$ bias on holder (a), (III) at $0 \mathrm{~V}$ bias on holder (b) and (IV) at $-100 \mathrm{~V}$ bias on holder (b).

bonding and cluster size can be obtained by this analysis. Figure 2 shows Raman spectra of DLC grown in holders (a) and (b) under similar deposition conditions, respectively. The spectra for samples in holder (a) show broad peaks appearing at $1350 \mathrm{~cm}^{-1}$ ( $D$ band) and $1580 \mathrm{~cm}^{-1}$ ( $G$ band). From the spectra, it is clear that the grown films contain both $s p^{2}$ and $s p^{3}$ bonded carbon, furthermore, as the peaks are relatively broad, the cluster size may be very small. Whereas for sample in holder (b), we observe only a broad $D$ band shifted to $1360 \mathrm{~cm}^{-1}$. It has been reported that $D$ band shifts to higher wavenumber with increasing disorder. This may be due to the presence of finite crystallite sizes from pyrolytic graphite, glassy carbon and carbon powders and due to $s p^{3}$ bonded species (Pandey and Patil 2007; Nakazawa et al 2008). The reason for absence of $G$ peak in sample in holder (b) is not clear.

To further understand the structure of DLC films, FTIR and UV-visible spectra were collected. The infrared spectra of DLC film deposited at 0 to $-175 \mathrm{~V}$ bias is shown in figure 3. Spectra below $3000 \mathrm{~cm}^{-1}$ refers to $s p^{3}$ bonded $\mathrm{CH}_{x}(x=1,2,3)$ which contributes atleast seven different vibrations. Some sharp IR absorption bands, in addition to the broad band that appears in the range $2880-3100 \mathrm{~cm}^{-1}$, indicate that the film has a complex $a-\mathrm{C}$ matrix formation. There is a strong absorption peak at $2955 \mathrm{~cm}^{-1}\left(\mathrm{CH}_{3}\right.$ groups $)$ with additional band at $2890 \mathrm{~cm}^{-1}\left(\mathrm{CH}_{2}\right.$ groups) showing the possibility of more ordered polymer like structure (Kulisch et al 2006). The band between $1700-1500 \mathrm{~cm}^{-1}$ and 1500 $1000 \mathrm{~cm}^{-1}$ shows absorptions from infrared active doublebonded and single bonded carbon groups, respectively. From the FTIR spectra, it seems that the film is polymeric in nature, and the polymeric nature is enhanced in case of samples of holder (b) in comparison to holder (a).

The UV-VIS transmittance spectra of the DLC films deposited on quartz substrates at different substrate bias are shown in figure 4 . It can be seen that high transmission ( $T>90 \%$ ) occurs in the range of $400-900 \mathrm{~nm}$. It is observed that the absorption in the UV region decreased with increase in bias. This may be due to the increase in $s p^{3}$ content with increasing bias. It is also observed that the transmittance increased with increasing bias. It may be due to the decrease in thickness of the films with increase in bias. The measured optical transparency ( $\sim 90 \%$ ), as shown in figure 5, further confirms it as polymeric type DLC (Robertson 2008). From the Raman, FTIR and UV-visible spectra that prove the presence of highly hydrogenated $s p^{3}$ polymeric carbon, it seems the grown films on both holders (a) and (b) result in polymeric DLC.

FESEM has been used to study the surface morphology and cross-section of DLC films; the cross-section images were used to measure film thickness. The films surface (image not shown) reveals featureless smooth surface 
morphology, which shows that the DLC films are of good quality having no detectable mechanical imperfections. Till date $a-\mathrm{C}$ films are not fully commercialized, due to the poor adhesion to substrates, which is caused mainly by high residual stresses in the films and high diffusion of carbon into the metal substrate (i.e. steel substrate) (Charitidis 2010). In figure 5, the FESEM cross-section images of the DLC films deposited on Si can be seen. With increase in bias from $0-100 \mathrm{~V}$, in case of holder $A$, the film thickness decreases from 70 to $30 \mathrm{~nm}$, whereas the film thickness in case of holder $B$ decreases from 300 to $40 \mathrm{~nm}$. In addition, it was observed that the films on the samples of holder $B$ at low bias $(<-50 \mathrm{~V})$ were powdery. For the same bias voltages, the change in thickness of the films deposited in bowl $B$ in comparison to bowl $A$, may be due to the high residence time of the depositing species as the radius of bowl $B$ is small and has steeper sidewall slope, however, this effect is minimal when $-100 \mathrm{~V}$ bias is used. The negative substrate bias (by energetic bombardment of positive ions) helps in deposition of a more compact and smooth film.

Hardness of DLC films was measured using nanoindentation system. Indentations were made by using a Berkovich diamond tip and Oliver-Pharr (1992) method was used for calculation of the hardness values. For measurement of hardness, the objective is to obtain a fully developed plastic zone within the film. Depending on the film/substrate properties, thickness of the film and radius of the indenter, this may not be possible. So usually a plot of hardness $(H)$ vs penetration depth is made and if there is a plateau in the values of $H$, then this is taken as the hardness of the film. It is generally observed that an influence from the substrate is negligible if the penetration depth is less than $10 \%$ of the film thickness, since the grown DLC films are $<300 \mathrm{~nm}$, so for these tests, very low load ranging from $0 \cdot 1-1 \mathrm{mN}$ was chosen so that the measured hardness value would not be affected much by the Si substrate. Figure 6 shows hardness of DLC films as a

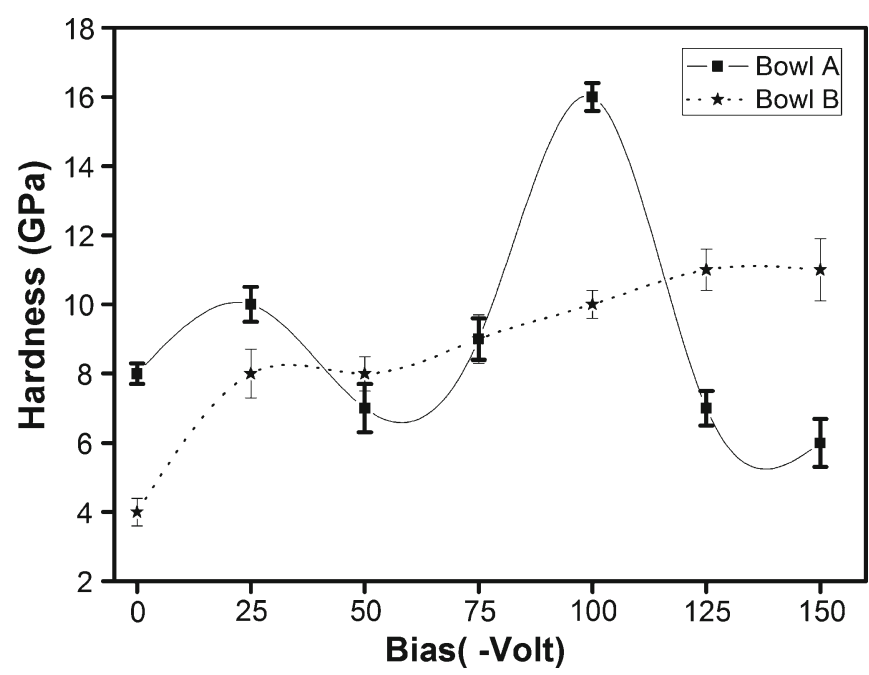

Figure 6. Hardness vs bias plot of DLC coated silicon substrate. function of substrate bias. At $-100 \mathrm{~V}$ bias, the sample on holder (a) exhibits higher hardness than the sample on holder (b). Even though the $s p^{3}$ content of the sample of holder (b) is more (from FTIR data), from the low nanoindentation hardness values it further proves that it has more of polymeric $\mathrm{C}-\mathrm{H} s p^{3}$ bonds which makes it soft.

\section{Conclusions}

At high pressure and low temperature, polymeric DLC films have been grown on two different sizes of bowl shaped steel substrate holder in a MWPACVD reactor without ECR. The films have smooth surface morphologies and polymeric structure. The polymeric nature of DLC is established by correlating Raman and FTIR spectra, visible transmittance data. Though the DLC films are polymeric, it is observed that appropriate choice of substrate holder (larger size) and bias $(-100 \mathrm{~V})$ helps in deposition of moderately hard films by partly overcoming the quenching and recombination effects (which usually occurs in the plasma at higher pressure and results in poorer coating). Our experimental method, having much simple vacuum design of the deposition system, can be used to deposit dense and scratch resistant anticorrosive polymeric DLC coatings. Elaborate scratch testing of DLC films grown are under progress which will be discussed in a future paper.

\section{Acknowledgement}

Financial support provided by DST for this work is acknowledged.

\section{References}

Achard J, Silva F, Tallaire A, Bonnin X, Lombardi G, Hassouni K and Gicquel A J 2007 Phys. D. Appl. Phys. 406175

Charitidis C A 2010 Int. J. Refract. Met. H. 2851

Choy K L 2003 Prog. Mater. Sci. 4857

Geis M W, Efremow N N, Krohn K E, Twichell J C and Lyszcazrz T M 1998 Nature 393431

Holland L and Ojha S M 1979 Thin Solid Films 58107

Kulisch W, Popov C, Boycheva S, Jelinek M, Gibson P N and Vorlicek V 2006 Surf. Coat. Technol. 2004731

Nakazawa H, Katoh S, Asai Y and Mashita M 2008 Jpn. J. Appl. Phys. 47231

Neuville S 2002 Diam. Relat. Mater. 111721

Neuville S and Matthews A 2007 Thin Solid Films 5156619

Oliver W C and Pharr G M 1992 J. Mater. Res. 71564

Pandey M and Patil D S 2007 Diam. Relat. Mater. 161912

Robertson J 2002 Mater. Sci. Eng. R37 129

Robertson J 2008 Phys. Status Solidi 2052233

Sails S R, Gardiner D J, Bowden M, Savage J and Rodway D 1996 Diam. Relat. Mater. 5589

Sumant A V, Gilbert P U P A, Grierson D S and Konicek A R 2007 Diam. Relat. Mater. 16718

Taniguchia M, Nagaoa H, Hiramatsub M, Andoa Y and Hori M 2005 Diam. Relat. Mater. 14855 